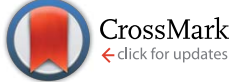

Cite this: RSC Adv., 2015, 5, 44074

Accepted 11th May 2015

DOI: 10.1039/c5ra02306h

www.rsc.org/advances

\section{Stimuli-responsive microgel-based etalons for optical sensing}

\author{
Yongfeng Gao, Xue Li and Michael J. Serpe* \\ Responsive polymer-based materials (or smart materials) have found numerous uses over the years due to \\ their ease of synthesis and various responsivities/functionalities. Of them, stimuli-responsive hydrogel \\ particles (microgels) have generated a lot of interest, and have been used for a number of applications, \\ most importantly for this submission is their use as components of photonic materials. In this review, we \\ highlight a few key examples of the use of stimuli-responsive materials for sensing applications, with a \\ particular focus on our work with microgel-based etalons.
}

\section{Introduction}

Stimuli-responsive polymers have the ability to respond physically and/or chemically to changes in their environment. ${ }^{1-3}$ These changes (or stimuli) can be $\mathrm{pH}$, temperature, ionic strength, light, electric or magnetic field. ${ }^{4-6}$ Ideally, the polymer response should be fully reversible upon the removal of the stimulus. While there are a number of polymers that respond to a variety of stimuli, thermoresponsive $\operatorname{poly}(\mathrm{N}$-isopropylacrylamide) (pNIPAm) is one of the most completely studied and well understood..$^{7-9}$ pNIPAm is fully water soluble at $T<32{ }^{\circ} \mathrm{C}$, existing as a random coil; pNIPAm transitions to a collapsed, "water insoluble" globule when the water is heated $>32{ }^{\circ} \mathrm{C} ; \mathbf{i}^{10,11}$ this transition is fully reversible over many cycles. ${ }^{12-14}$ The conformational change is also accompanied by water exchange process. That is, when pNIPAm undergoes the coil to globule transition, water is expelled, while water is "sorbed" when the polymer undergoes the opposite process.

Hydrogels, which are water swollen polymer networks, can be readily synthesized. ${ }^{15-17}$ Additionally, hydrogel particles can be generated, and can be made to have diameters ranging from tens of nanometers to several micrometers; this depends on the synthetic conditions. ${ }^{12,14,18-22}$ Hydrogel particles are referred to as nanogels or microgels depending on their diameter; typically nanogels have diameters $<100 \mathrm{~nm}$, while microgels have larger diameters. Stimuli responsive microgels/nanogels have emerged as important moieties for sensing, ${ }^{23-25}$ chemical separation, ${ }^{26,27}$ and drug delivery. ${ }^{28-30}$ Microgels/nanogels that are responsive to temperature, ${ }^{12,14,20,31} \mathrm{pH},{ }^{32,33}$ magnetic field, ${ }^{34-36}$ electric field, ${ }^{37}$ metal, ${ }^{38,39}$ small molecules ${ }^{40-42}$ and ionic strength ${ }^{43}$ have been synthesized. Additionally, individual nanogels/microgels can be made responsive to multiple stimuli by simply adding monomers that yield the desired combined

Department of Chemistry, University of Alberta, Edmonton, AB, T6G 2G2, Canada. E-mail: michael.serpe@ualberta.ca response/chemical functionality during the time of synthesis. For example, it has been shown that pNIPAm microgels can be made $\mathrm{pH}$ responsive by adding, e.g., acrylic acid (AAc), ${ }^{36}{ }^{2-}$ hydroxyethyl methacrylate, ${ }^{44} \quad N$-[3-(dimethylamino)propyl]methacrylamide ${ }^{45}$ and 3-(acrylamido)phenylboronic acid. ${ }^{46}$ Interestingly, the resultant microgels maintain the thermoresponsivity of the pNIPAm, with the additional responsivity of the comonomer, rendering the microgels multiresponsive.

As mentioned above, stimuli responsive hydrogels/microgels have been used for a variety of applications; among those applications, their use as optical sensors is most important for the current submission. One early example of the use of hydrogels for optical sensing was presented by Asher and coworkers, and involved entrapping a colloidal crystal in a responsive hydrogel network. ${ }^{47-49}$ Specifically, they showed that colloidal particles that exhibited long-range order (a colloidal crystal) could be held into place, and their structure retained, by polymerizing hydrogels of various compositions in the interstices between the colloids. The materials exhibited bright color, which depended on the colloidal crystal's lattice spacing, which could be tuned by the hydrogel solvation state. This behavior is shown schematically in Fig. 1. The Asher group demonstrated in many publications that their materials could be used to detect a variety of analytes, including metal ions and a variety of other small molecules including humidity and ammonia, ${ }^{50}$ surfactant, ${ }^{51}$ glucose,${ }^{52}$ organophosphates, ${ }^{53}$ and ethanol. ${ }^{54}$ In one specific example, Asher and coworkers ${ }^{55}$ prepared a novel protein-based hydrogel photonic crystal, which acts as a coulometer (shown in Fig. 2), whose volume is very sensitive to the net charge on the protein-based structure, which can be related to analyte binding. The resulting volume changes shift the diffraction of a two dimensional (2D) array attached to the protein hydrogel surface. The Asher group showed that the charged state of the hydrogel could be related to the presence (and concentration) of the analytes salicylate, ibuprofen and picosulfate. 

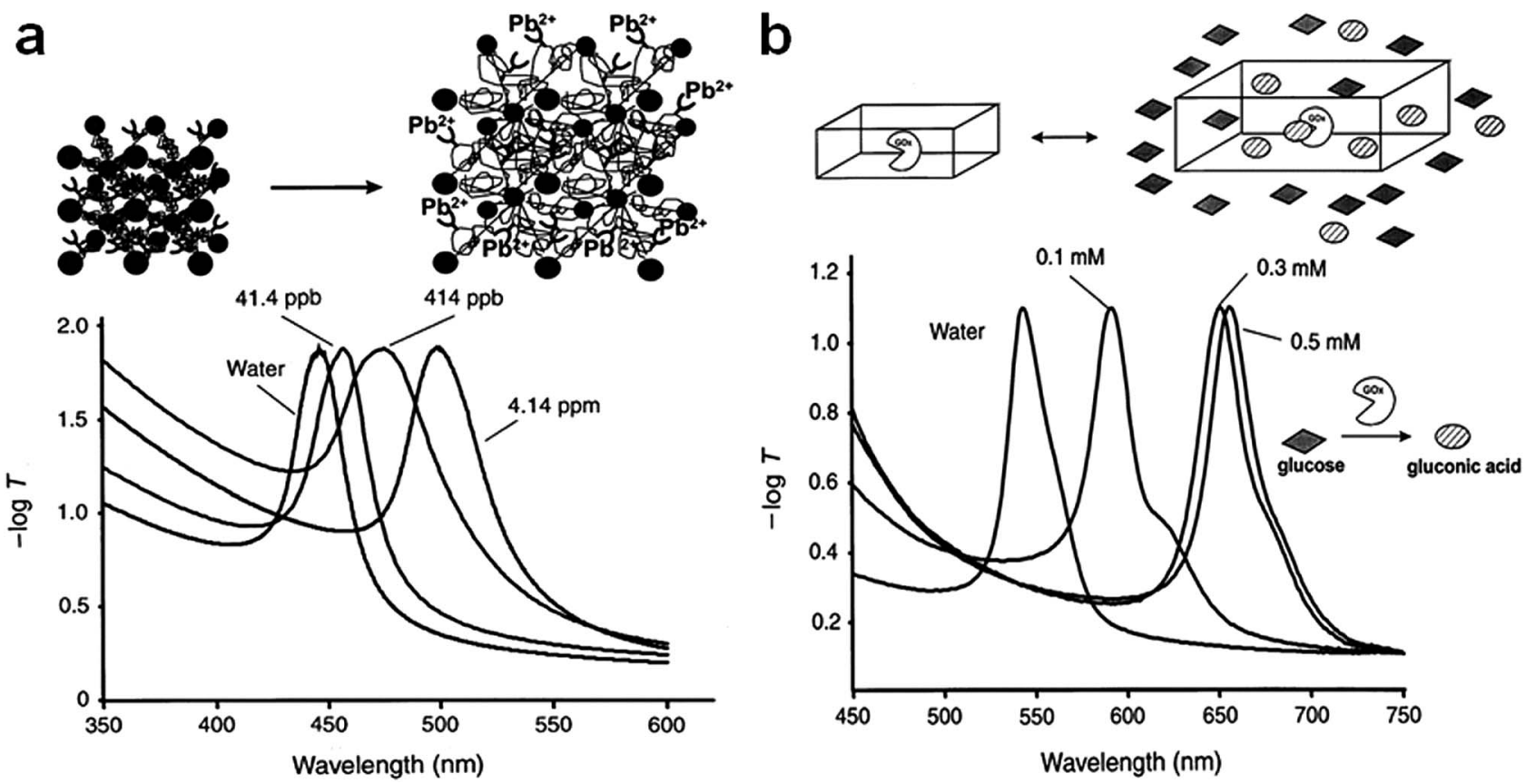

Fig. 1 (a) Visible extinction spectra of a hydrogel based $\mathrm{Pb}^{2+}$ sensor at various concentrations of $\mathrm{Pb}\left(\mathrm{CH}_{3} \mathrm{COO}\right)$ and (b) spectra showing how diffraction depends on the glucose concentration. Reprinted with permission from ref. 47, Copyright 1997, Nature publication.

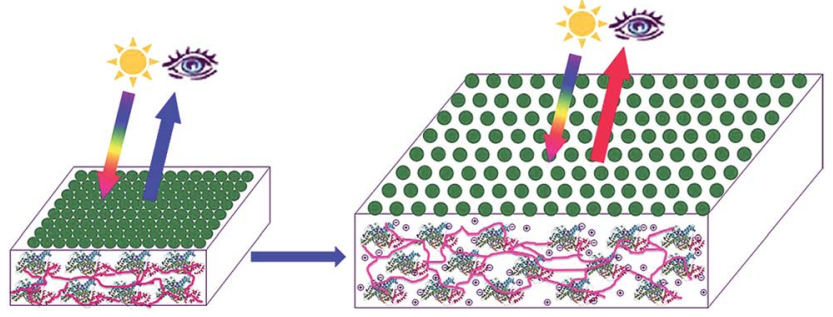

Fig. 2 The binding of charged species to a protein hydrogel gives rise to Donnan potentials that change the hydrogel volume causing shifts in the lattice spacing and the subsequent diffraction. Reprinted with permission from ref. 55, Copyright 2014, American Chemistry Society.

There are many other recent examples in the literature describing a wide variety of responsive photonic materials systems for sensing applications. ${ }^{56-60}$ In one example, Wang and co-workers $^{60}$ developed a polyacrylamide (PAAm) hydrogelbased photonic material that exhibited specific visual colors at various humidity. In another example, Hamad and MacLachlan $^{58}$ reported a novel photonic material prepared with nanocrystalline cellulose, which can undergo rapid and reversible changes in color upon swelling or pressing, and potentially can be applied to biosensing, unique responsive optics and so on. Finally, in another example, Hayward and coworkers $^{56}$ proposed a method to develop a one dimensional (1D) photonic multilayer colorimetric sensor based on photocrosslinkable copolymer films. The photonic material was created by sequentially depositing layers of high- and lowrefractive index photo-crosslinkable copolymers on a substrate, like glass slides. The system offers great flexibility for tailoring the optical properties by depositing polymers that exhibit specific responsivities, for example, to generate electric field responsive multilayers a conductive copolymer could be used.

Many reviews have been written that highlight the multiple ways photonic materials can be used as optical sensors, ${ }^{61-64}$ and will not be rehashed here. The remaining manuscript will be devoted to describing pNIPAm microgel-based etalons. Photonic materials fabricated by a number of groups ${ }^{65-68}$ have refractive index order periodicity in 1, 2, and 3 dimensions (1D, $2 \mathrm{D}$, and $3 \mathrm{D}$, respectively). Materials used for constructing these kinds of structures could be nanoparticles, polymer beads, hydrogels and so on. In our previous studies and reviews, ${ }^{61,62,69}$ we introduced the basic etalon concept and how we constructed pNIPAm microgel-based 1D photonic material structures. ${ }^{61,62}$ In this submission, we review our recent work with this optical device.

\section{Microgel-based etalons}

The Serpe Group developed pNIPAm microgel-based photonic materials (etalons) and has extensively investigated their optical

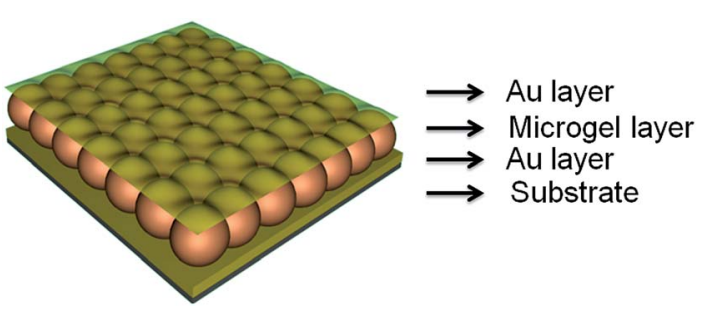

Fig. 3 Structure of microgel-based etalons. 


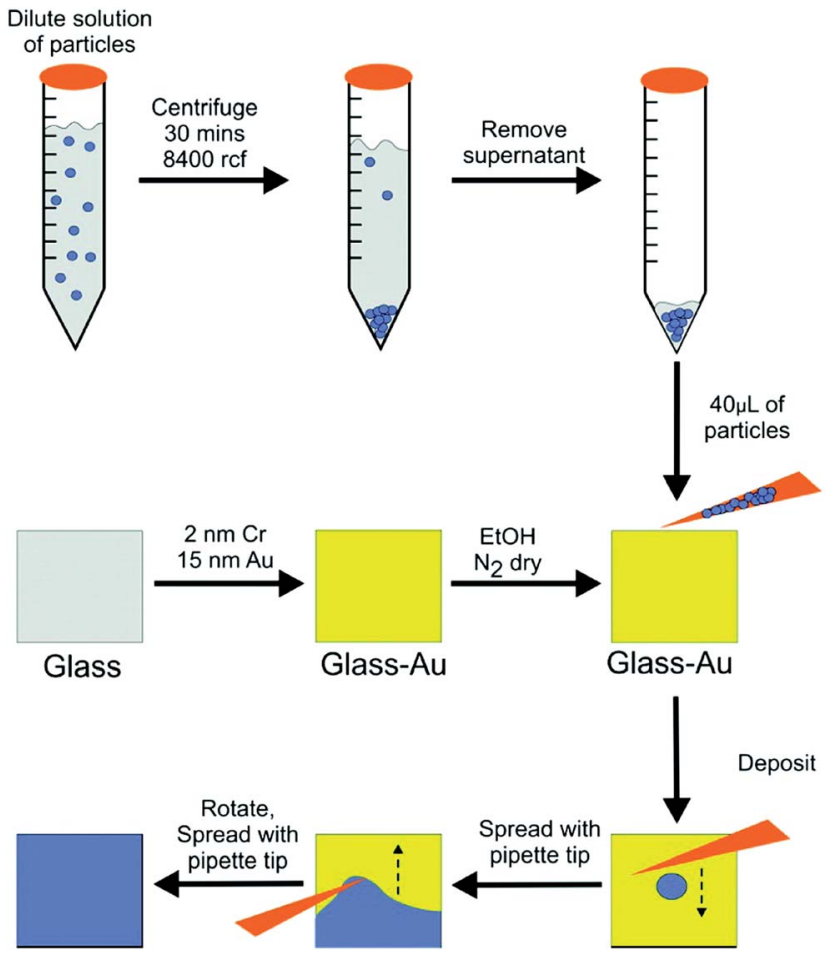

Fig. 4 The "paint-on" protocol developed for microgel-based etalon fabrication. Reprinted with permission from ref. 80, Copyright 2012, American Chemistry Society.

properties and behavior. Furthermore, many investigations were undertaken and we have many published examples demonstrating their utility as chemical/biological sensors. ${ }^{\mathbf{4 2 , 7 0 - 7 4}}$ Microgel-based etalons have a very simple structure, which are composed of two reflective, semitransparent surfaces (typically metal) sandwiching a microgel layer. The standard structure of etalons fabricated in our lab ${ }^{64,73,75-78}$ is shown schematically shown in Fig. 3. These devices contain two thin Au layers sandwiching a "monolithic" monolayer of microgels all supported on a glass substrate. This structure allows light to enter the dielectric cavity and resonate between the two reflective layers. This resonating light yields constructive and destructive

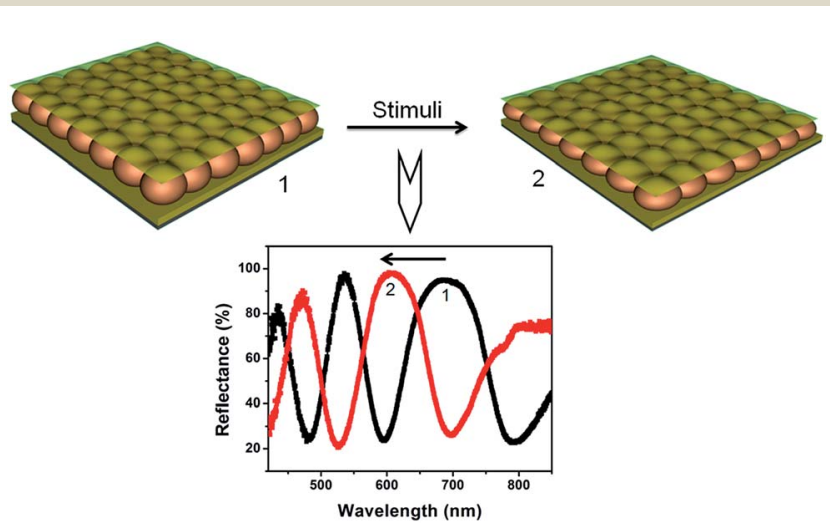

Fig. 5 Microgel-based etalon's response to an external stimulus, which yields a shift in the peaks of the reflectance spectrum.

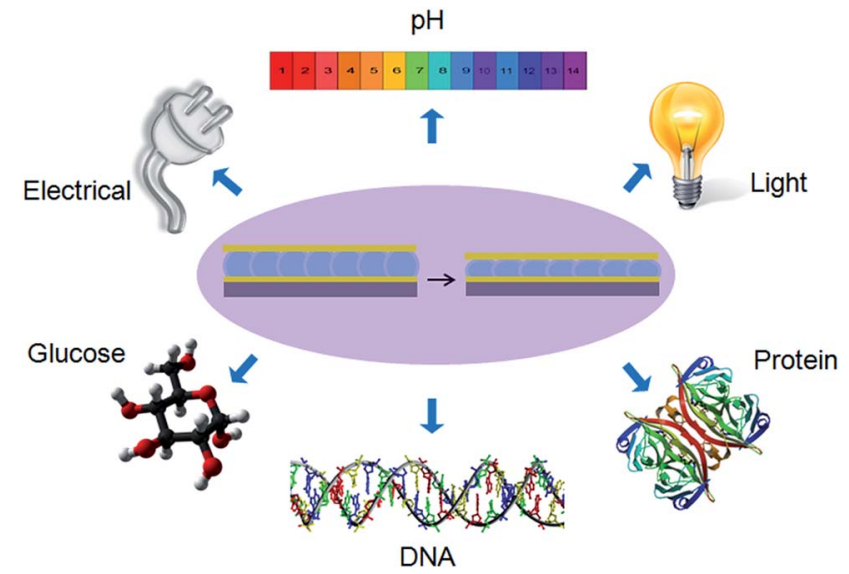

Fig. 6 Stimuli that are able to yield an optical response from a microgel-based etalon.

interference, allowing certain wavelengths of light to be reflected. The wavelength of the reflected light can be calculated using eqn (1):

$$
\lambda m=2 n d \cos \theta
$$

where the specific wavelength maximum of the peak $(\lambda)$ depends on the peak order $(m)$, refractive index of the dielectric $(n)$ and the spacing between the mirrors $(d)$, as well as the angle of incidence $(\theta)$. In our etalons, Au and pNIPAm-based microgels serve as the mirrors and the dielectric layer, respectively.

a

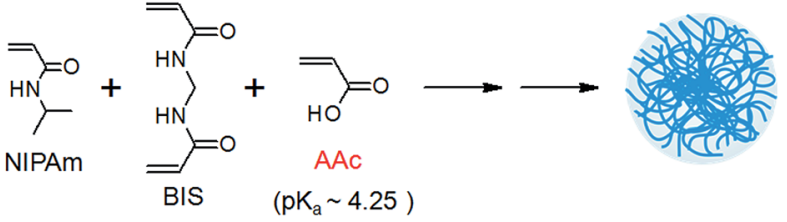

b

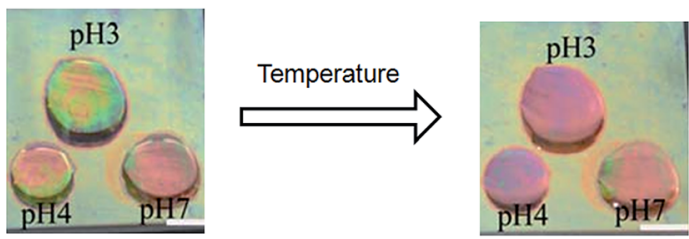

C

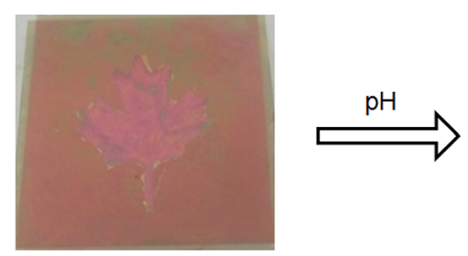

Fig. 7 (a) Schematic showing the synthesis of pNIPAm-CO-AAc microgels, that yield $\mathrm{pH}$ responsive etalons. (b) Temperature responsivity of pNIPAm-co-AAc microgel-based etalons at different $\mathrm{pH}$ values. (c) Maple leaf pattern composed of pNIPAm-cO-AAc microgels change color when the solution $\mathrm{pH}$ is varied from $\mathrm{pH} 3.0$ and (right) 6.5. Reproduced with permission from ref. 73, Copyright 2014, Royal Society of Chemistry. 


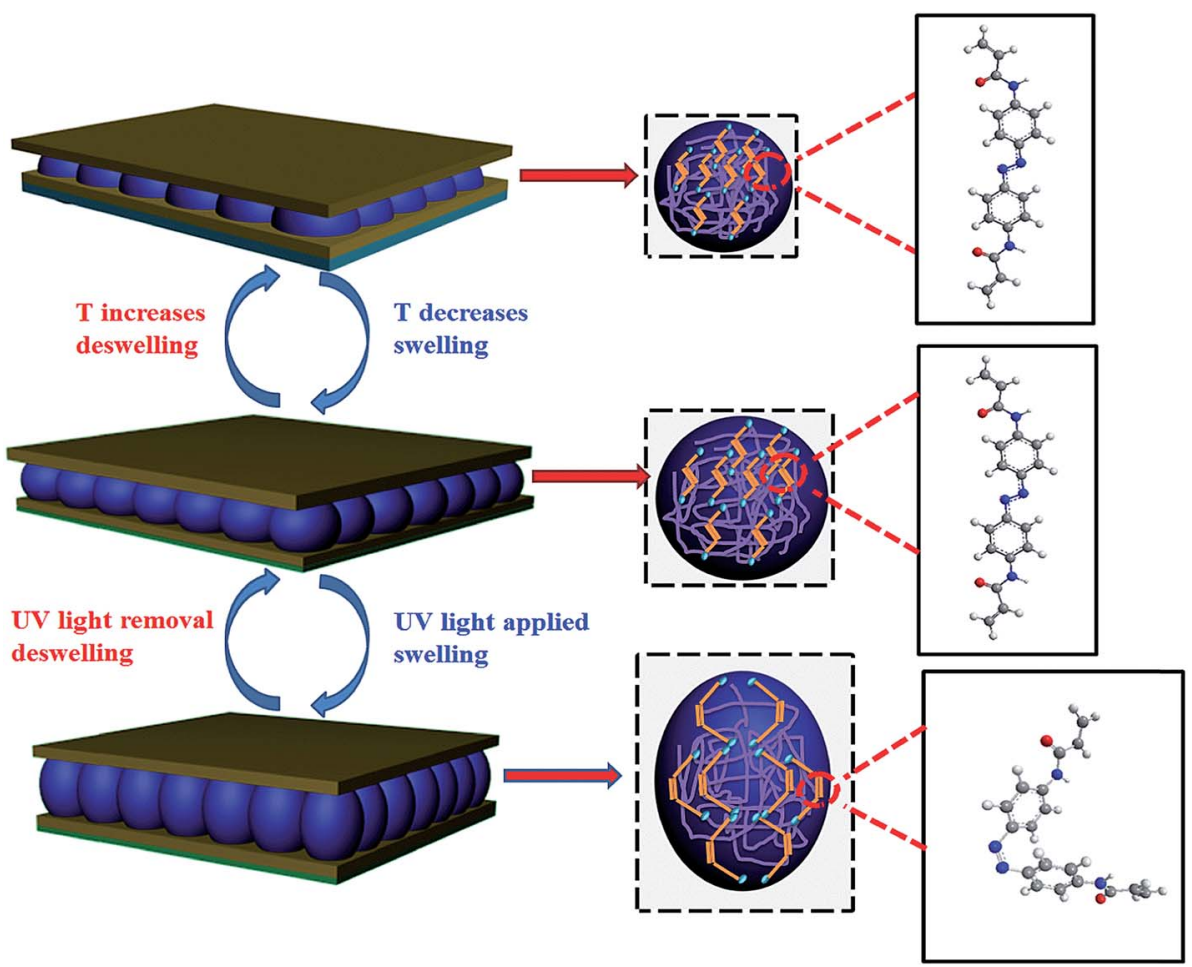

Fig. 8 Light responsive etalons. The structure of azobenzene can be switched by exposure to light. Specifically, UV exposure causes the azobenzene to transition to the cis conformation, which is fully reversible back to the trans conformation. This behavior led to microgel solvation state changes, and a change in the optical properties of the etalons. Reproduced with permission from ref. 84, Copyright 2014, Royal Society of Chemistry.

a<smiles>O=Cc1ccccc1[N+](=O)[O-]</smiles>
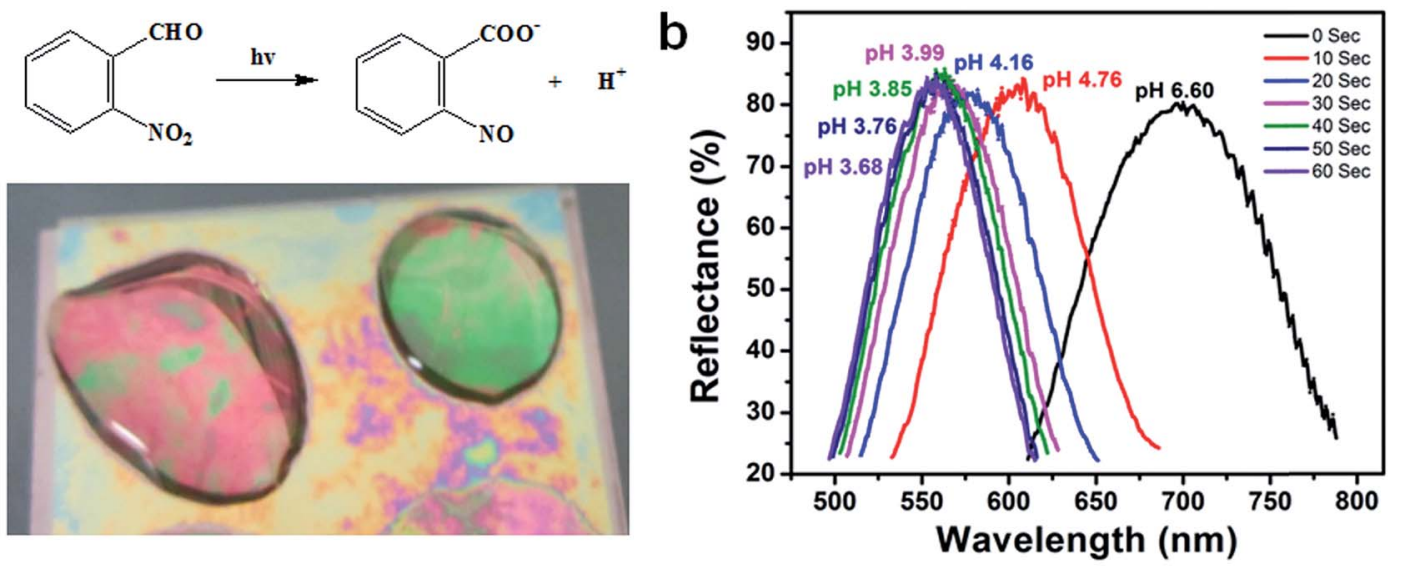

C
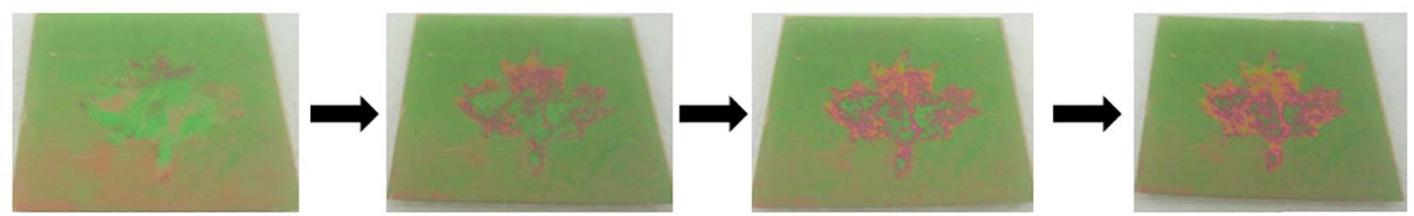

Time
0 min

$1 \mathrm{~min}$

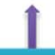

$2 \mathrm{~min}$

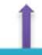

$3 \mathrm{~min}$

Fig. 9 (a) Schematic depiction of the photoacid generation process upon exposure to UV light, and a pH responsive etalon that changes color upon acid generation. (b) The dependence of the device reflectance spectrum at different pH values that were changed by UV exposure. (c) Photographs of patterned etalons (maple leaf is $\mathrm{pH}$ responsive) after the indicated irradiation times. Reproduced with permission from ref. 83, Copyright 2014, Royal Society of Chemistry. 


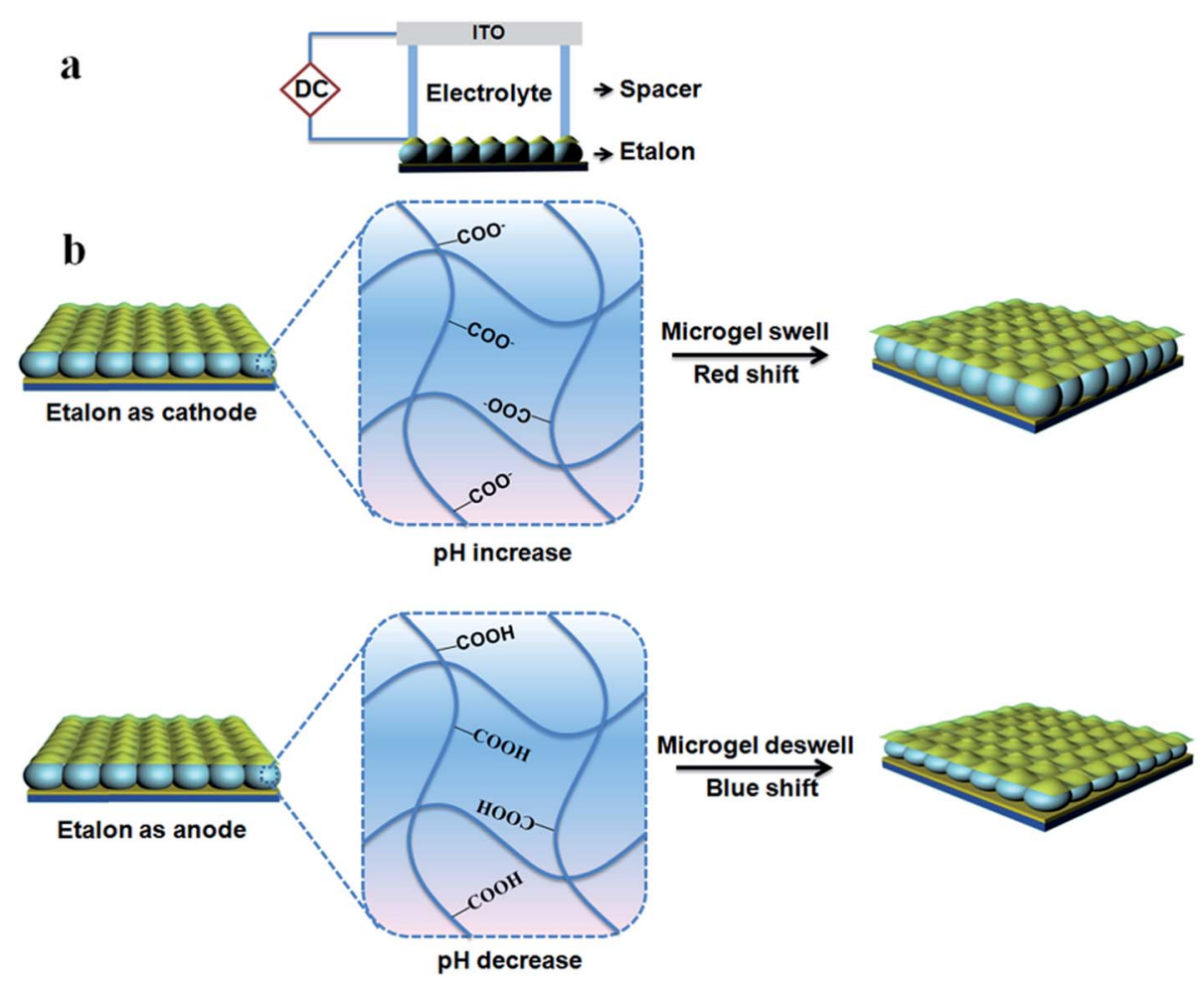

Fig. 10 (a) Schematic of the experimental setup. (b) Schematic depiction of the system's response to the application of a potential, which changes the solution $\mathrm{pH}$ yielding a device response. Reproduced with permission from ref. 85, Copyright 2014, Royal Society of Chemistry.

\subsection{Fabrication of microgel-based etalons}

pNIPAm microgel-based etalons were fabricated by thermally evaporating layers of $2 \mathrm{~nm} \mathrm{Cr}$ and $15 \mathrm{~nm}$ Au on a glass substrate (other substrates can and have been used). ${ }^{79}$ Following this step, pNIPAm-based microgels were coated on the Au layer using our developed "painting" technique, ${ }^{80}$ which is shown in schematically Fig. 4. To accomplish this, a $40 \mu \mathrm{L}$ aliquot of concentrated microgels was deposited onto a Au substrate at $30^{\circ} \mathrm{C}$ and spread toward each edge using the side of a micropipette tip until the microgels covered the entire Au substrate. The spreading continued until the microgel solution was too viscous to spread over the surface. At that point, the microgels were allowed to dry completely on the substrate for $2 \mathrm{~h}$ at $35^{\circ} \mathrm{C}$. After $2 \mathrm{~h}$, the dry film was rinsed with copious amounts of deionized (DI) water and soaked in DI water overnight to remove microgels not bound directly to the Au. This method yields an extremely uniform monolithic monolayer of microgels, on which an additional $2 \mathrm{~nm} \mathrm{Cr}$ and $15 \mathrm{~nm}$ Au were deposited on the microgel layer. We point out that the painting protocol can be applied to coat microgels with a variety of functionalities on substrates composed of a variety of metals. ${ }^{79}$ Using this approach, we also showed that pNIPAm microgel-based etalons could be generated from various metals, including $\mathrm{Al}, \mathrm{Cu}, \mathrm{Ni}$, and $\mathrm{Ti}^{79}$

\subsection{Optical properties of microgel-based etalons}

Reflectance spectroscopy, as well as visual color, was used to investigate the optical properties and responsivity of the microgel-based etalons. Fig. 5 shows a typical reflectance spectrum from our devices. As can be seen, the spectrum exhibits multiple peaks, positioned at specific wavelengths, which are defined primarily by the thickness of the pNIPAm microgel-based layer (the microgel layer refractive index does play a small role). Most interesting is the ability of the peaks to shift to different wavelengths as a stimulus is applied to the etalon. The most basic response of our devices is to temperature. At high temperature, the pNIPAm microgel-based layer collapses, which brings the etalon's metal layers close to one another. ${ }^{75,76,78}$ This necessarily shifts the position of the reflectance peaks to lower wavelengths, according to eqn (1). In our previous studies, we prepared numerous stimuli responsive microgel-based etalon devices, and studied their behavior. These include devices responsive to: $\mathrm{pH}^{74,75,81,82}$ light, ${ }^{\mathbf{8 3} 84}$ electrical fields, ${ }^{85}$ glucose ${ }^{42}$ DNA, ${ }^{86,87}$ protein, ${ }^{63,70,71}$ macromolecules $^{72,88}$ and so on as shown in Fig. 6. Some of these applications will be detailed in the next section.

\section{Applications}

\section{1. pH sensing}

As pointed out above, color tunable microgel-based etalons can change their optical properties in response to a wide variety of stimuli. These responses have lead to many sensing applications. In one early study, ${ }^{73,77}$ we demonstrated that the etalons could be made responsive to solution $\mathrm{pH}$ by incorporation of weak acids/bases into the microgel structure via copolymerization during the time of microgel synthesis. Acrylic acid is commonly used in our lab, which exhibits a $\mathrm{p} K_{\mathrm{a}}$ of $\sim 4.25$. As a 


\section{a}

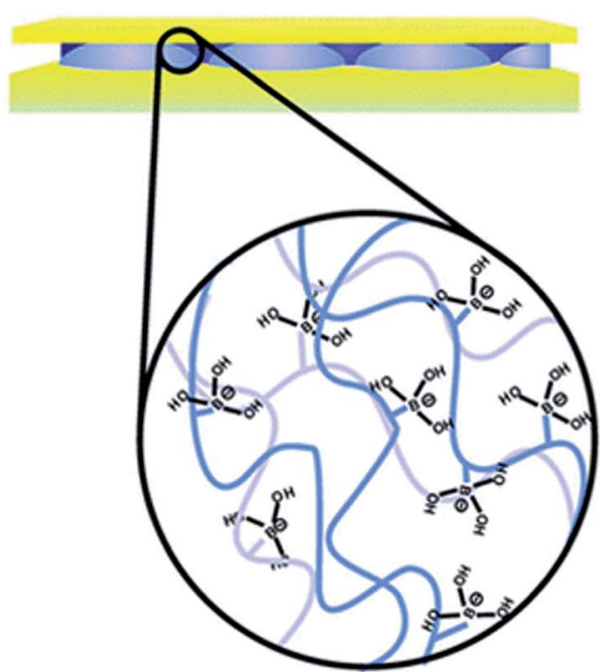

b

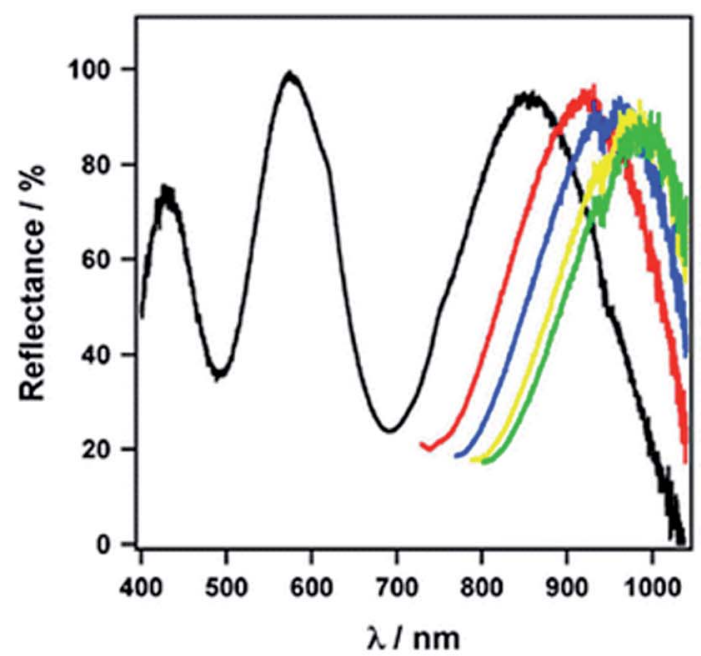

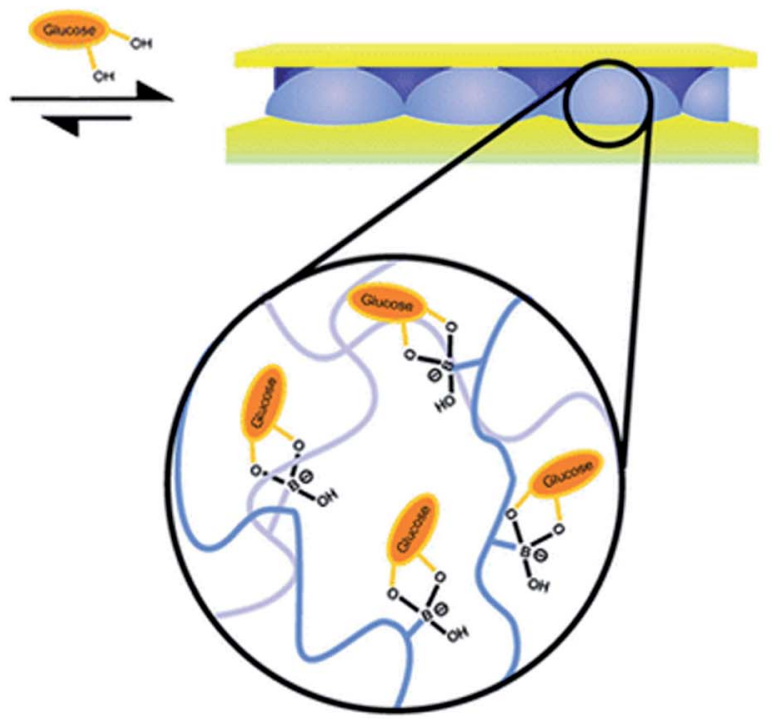

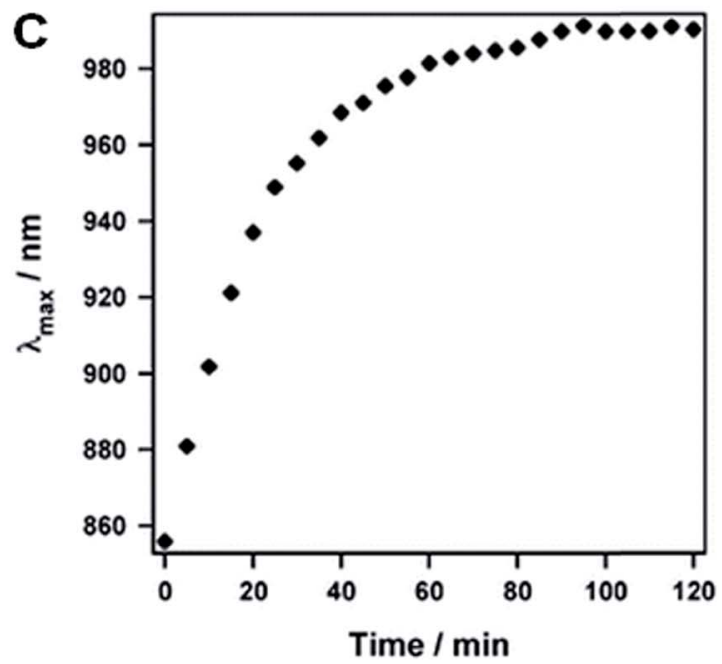

Fig. 11 (a) Schematic depiction of the glucose responsivity of an 3-aminophenylboronic acid (APBA)-functionalized microgel etalon at pH 9. Glucose responsivity shown as (b) a shift in the position of the peaks in the device's reflectance spectrum for the device composed of pNIPAmbased microgels modified with APBA. (c) Peak position as a function of time for the high wavelength peak in (b). For panel (c), the black trace is the initial spectrum, in addition to truncated spectra for the most red-shifted peak as a function of time after glucose introduction: (red) 15, (blue) 30, (yellow) 60, and (green) 120 min. Reprinted with permission from ref. 42, Copyright 2010, Springer.

result, the microgels become negatively charged, and swell at $\mathrm{pH}>\mathrm{p} K_{\mathrm{a}}$, leading to a concomitant change in the etalons optical properties. Specifically, the microgel swelling leads to an increase in the $\mathrm{Au}-\mathrm{Au}$ spacing, yielding a red shift in the device's reflectance peaks. We showed that the optical properties of spatially isolated regions of our etalon device can be changed independently in response to temperature and $\mathrm{pH},{ }^{77}$ as shown in Fig. 7. In another example, we showed that a patterned region of an etalon could be optically switched by changes in solution pH. Fig. 7 shows an example of a maple leaf that was composed of $\mathrm{pH}$ responsive pNIPAm-co-AAc microgels, while the surrounding etalon was composed of non-pH responsive pNIPAm microgels. After immersing this etalon device into different $\mathrm{pH}$ solutions, $\mathrm{pH} 3.0$ and $\mathrm{pH}$ 6.5, the maple leaf color was dramatically switched independent of the surrounding etalon. ${ }^{83}$ This behavior shows the sensitivity of etalons to solution $\mathrm{pH}$ changes and demonstrates its use as a $\mathrm{pH}$ sensor.

\subsection{Display devices}

Light responsive etalons were also prepared by fabricating etalons with azobenzene crosslinked microgels. Fig. 8 shows that ultraviolet (UV) light could be used to induce a cis-trans isomerization of the azobenzene molecule that is crosslinking the microgels, resulting in a change in the etalon's optical properties. ${ }^{\mathbf{8 4}}$ Etalon-based systems were also prepared that are capable of changing their optical properties in response to light by employing a photoacid combined with $\mathrm{pH}$ responsive microgels. Specifically, a photoacid is a molecule that is capable of generating protons when exposed to UV irradiation, which can decrease the $\mathrm{pH}$ of a solution. Fig. 9 shows the relationship 


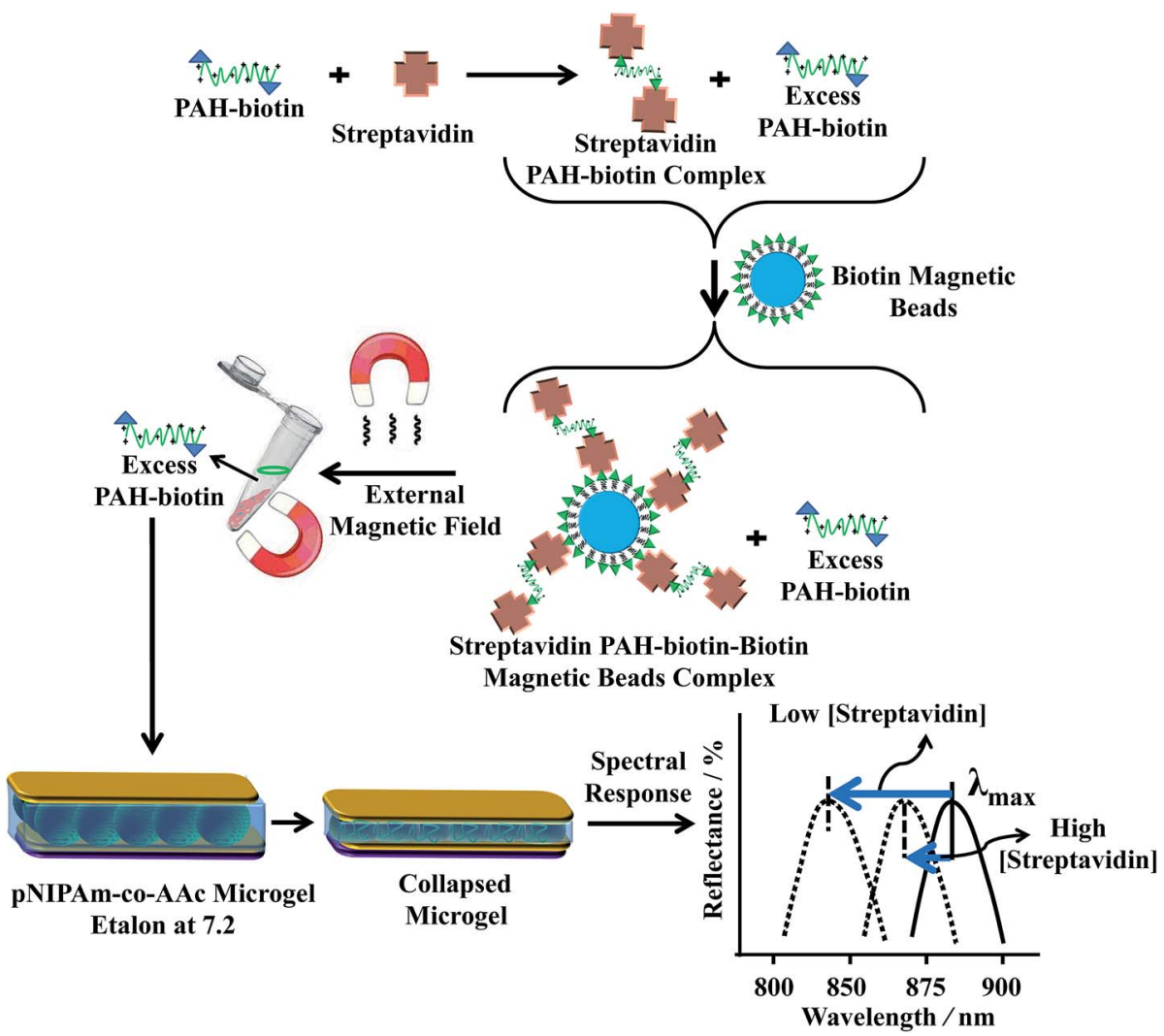

Fig. 12 Streptavidin (the analyte) is added to an excess amount of biotin-modified poly(allylamine hydrochloride) (PAH). The streptavidin-biotin$\mathrm{PAH}$ complex is then removed from solution using biotin modified magnetic particles, leaving behind free, unbound PAH. The unbound PAH is subsequently added to a pNIPAm-CO-AAc microgel-based etalon immersed in aqueous solution at a pH that renders both the microgel layer and the PAH charged. As a result, the etalon's spectral peaks shift in proportion to the amount of PAH-biotin that was added. This, in turn can be related back to the original amount of streptavidin added to the PAH-biotin. Reprinted with permission from ref. 71, Copyright 2014, Elsevier.

between UV irradiation times, pH change and wavelength shift that also yields a visible color change - these results were achieved using the photoacid $o$-nitrobenzaldehyde $(o$-NBA). The color of this device could be visibly changed in less than $3 \mathrm{~min}^{83}$ Light responsivity can easily be initiated/stopped by simply switching the excitation source on/off, while the magnitude of the response can be tuned by modulating the excitation source intensity, and/or wavelength.
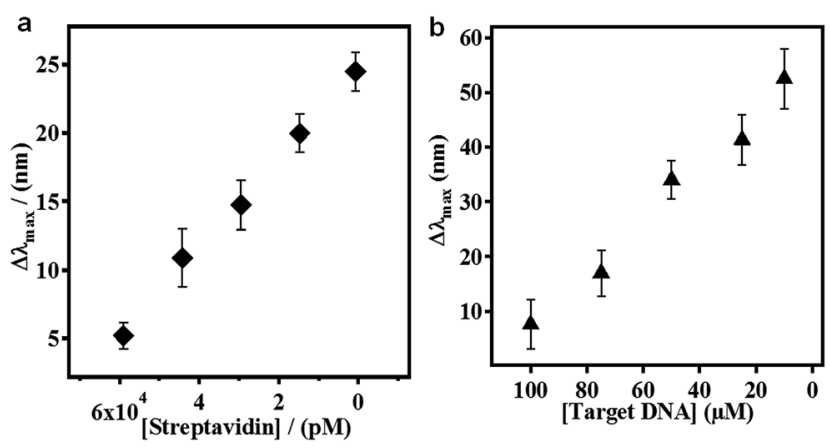

Fig. 13 (a) The etalon response to various streptavidin, and (b) TDNA concentrations. Each point represents the average of at least three independent measurements, and the error bars are standard deviation for those values. Reprinted with the permission of 71 Copyright 2014, Elsevier, and 86 Copyright 2014, Springer.
We also showed that etalons could be made responsive to the application of electric fields, as shown in Fig. $10 .{ }^{85}$ In this study, by applying a certain voltage $(\sim 3 \mathrm{~V})$, a $\mathrm{pH}$ responsive etalon could exhibit visible and reversible color change. This is due to the electrical potential hydrolyzing the water of the electrolyte solution, which subsequently changes its $\mathrm{pH}$. Hence, if $\mathrm{pH}$ responsive etalons are exposed to this system, the etalon optical properties will likewise depend on applied electrical potential. We show that the etalon's optical properties (color) are stable for many hours, until an appropriate potential is applied to bring the solution $\mathrm{pH}$ back to its initial value. In this example, we constructed an etalon with a maple leaf pattern that was $\mathrm{pH}$ responsive - the color of the maple leaf changed when an electrical potential was applied, and was reversible over many cycles.

\subsection{Glucose sensing}

Glucose sensitive microgels were also synthesized by modification of the microgels with aminophenylboronic acid (APBA). APBA-functionalized pNIPAm-based microgels have been shown to change solvation state in a manner that depended on glucose concentration. Specifically, they swell in the presence of glucose, as shown schematically in Fig. 11(a). Therefore, fabrication of etalons from these microgels should yield a material that changes color in response to glucose addition. ${ }^{42}$ As shown in 


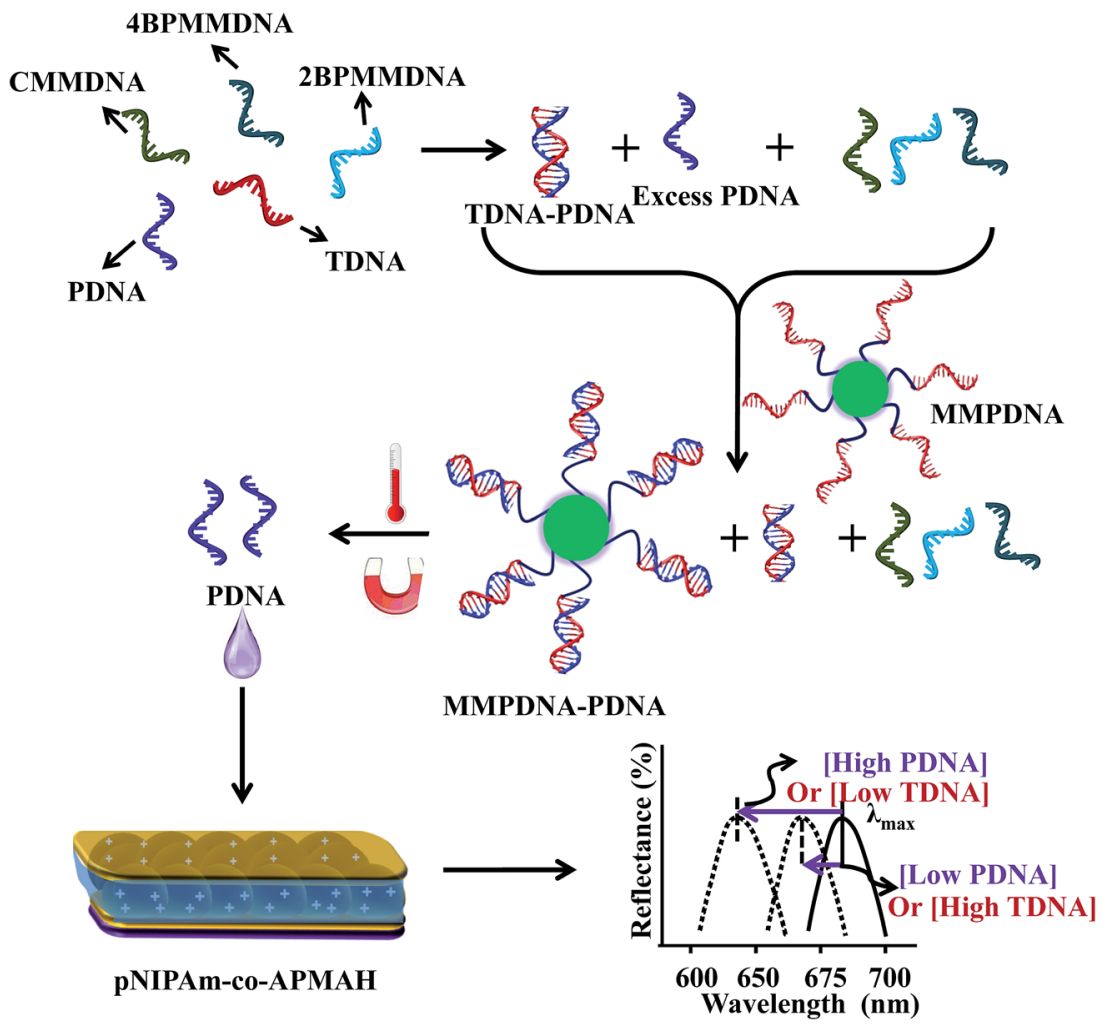

Fig. 14 The protocol used for indirectly sensing target DNA (TDNA), by sensing probe DNA (PDNA). An excess amount of PDNA is exposed to a solution containing TDNA and DNA with a completely mismatched sequence (CMMDNA), and DNA with four (4BPMMDNA) and two base mismatches (2BPMMDNA). The PDNA binds the TDNA completely, leaving behind excess, unbound PDNA in solution. Magnetic microparticles (MMPDNA) that are functionalized with the complete complement to PDNA are added to the solution to capture the excess PDNA. In this case, a large spectral shift from the etalon corresponds to a large excess of PDNA, which means a low concentration of TDNA was present in the initial solution. The opposite is true as well-a low concentration of PDNA left in solution yields a small spectral shift from the device, meaning there was a large amount of TDNA present in the initial solution. This illustrates the strength of the current system-low concentrations of TDNA yield large spectral shifts making the device more sensitive to low DNA concentrations. Reprinted with permission from ref. 86, Copyright 2014, Springer.

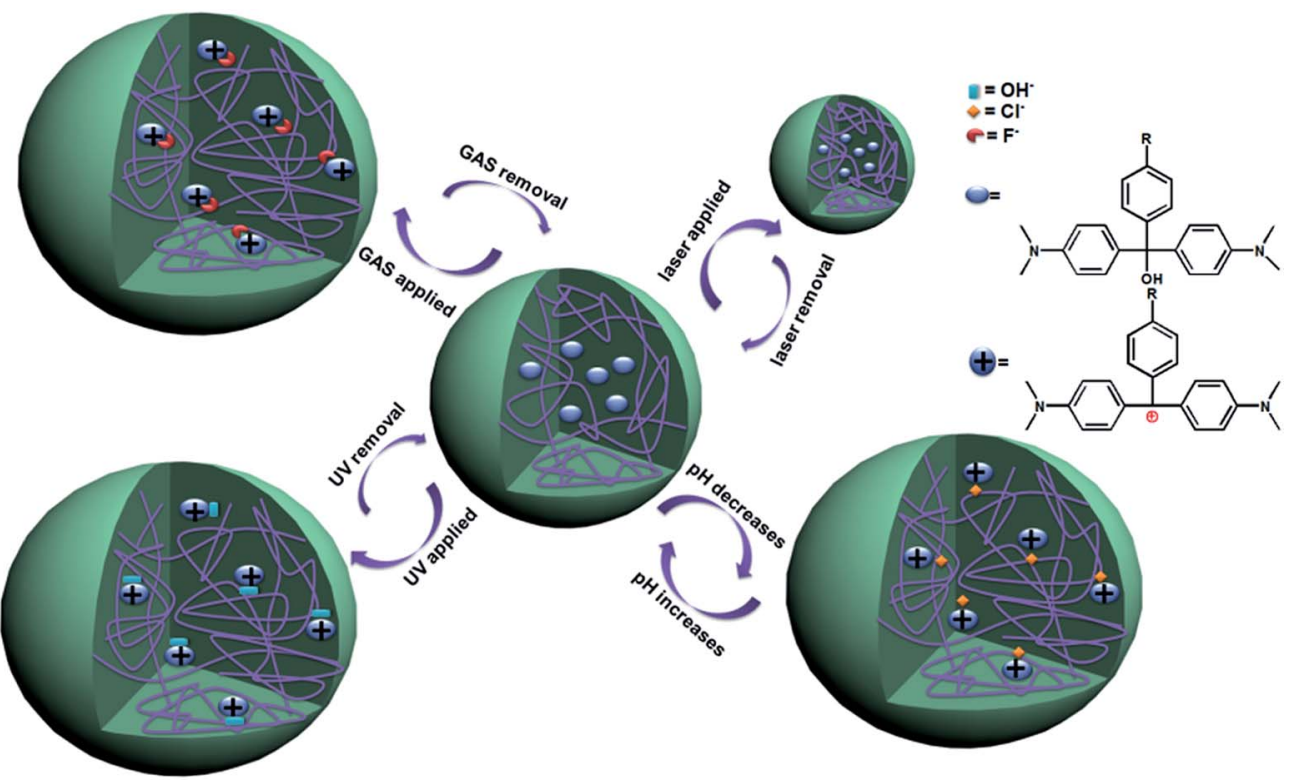

Fig. 15 The various responses expected from TPL-modified microgels. The TPL structure is shown on the top right. Reprinted with permission from ref. 89, Copyright 2014, WILEY-VCH Verlag GmbH \& Co. KGaA, Weinheim. 
Fig. 11(b) and (c), there is a significant spectral red-shift $(\sim 134 \mathrm{~nm})$ in the presence of $3 \mathrm{mg} \mathrm{mL} \mathrm{m}^{-1}$ solution of glucose (pH 9 carbonate buffer) and the majority of the spectral shift occurs within 30 minutes of glucose introduction, therefore yielding a visible color change. This result allowed us to conclude that etalons could potentially be used for detecting and quantifying biologically relevant molecules as well for biosensing purpose.

\subsection{Macromolecule sensing}

To move our technology forward, we showed that etalons could be used for sensing macromolecules, ${ }^{72,88}$ proteins $^{70,71}$ and DNA. $^{86,87}$ The protein sensing protocol is shown in Fig. 12. Initially, we established that the poly(allylamine hydrochloride) (PAH)-biotin could penetrate the etalon and crosslink the microgel layer leading to a detectable spectral shift. Once this was established, we showed that this approach could be used for detecting streptavidin, as can be seen in Fig. 13(a). ${ }^{70,71} \mathrm{We}$ found that the extent of the etalon's reflectance peak shift was linearly dependent on streptavidin concentration, over the range investigated. One unique feature of our devices is their ability to exhibit large responses to low analyte concentrations, which is opposite of most other analytical techniques that yield small responses in the presence of low analyte concentrations. Utilizing a similar approach, we also showed that etalons were able to detect specific DNA sequences in solutions. ${ }^{\mathbf{8 6 , 8 7}}$ The sensing procedure as can be seen in Fig. 14, micromolar concentrations of target DNA in solutions containing two and four base pair mismatch sequences was capable of detecting without the use of labels, and the data is shown in Fig. 14(b), the signal was linear response to the target DNA concentration and also, lower target concentration gives larger response.

\subsection{Multianalyte sensing}

More recent efforts have been focused on constructing individual etalons that are responsive to multiple stimuli. In a

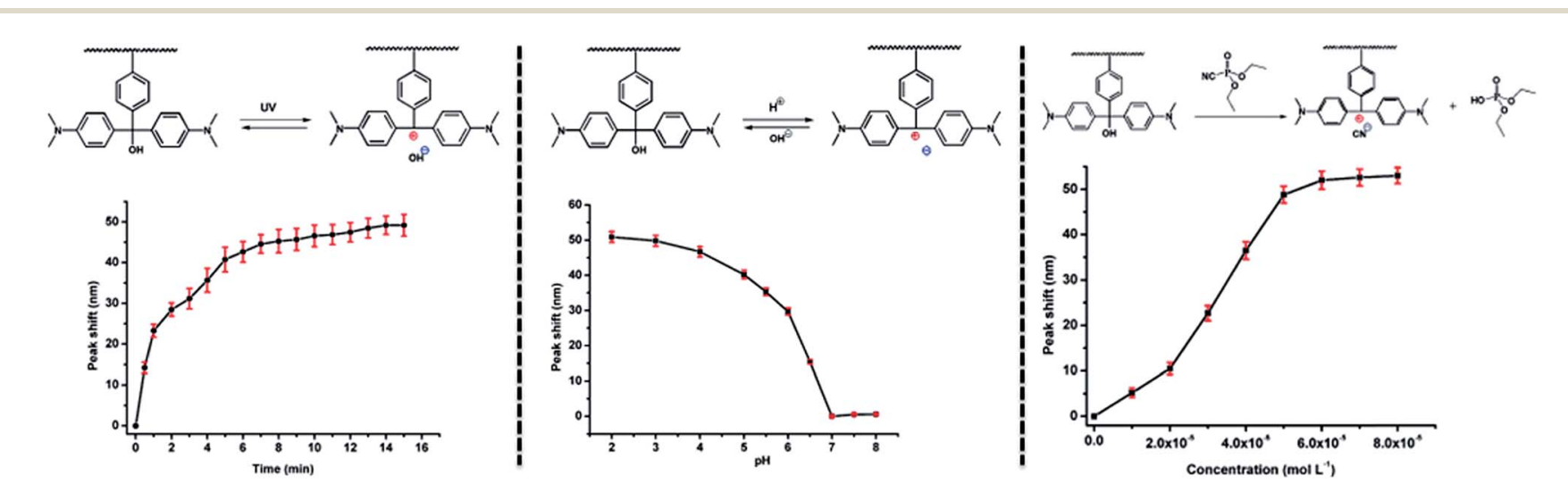

Fig. 16 Peak shifts for etalons composed of TPL-modified microgels; (left) their response to UV light; (middle) their response to pH; and (right) their response to GAS. Reprinted with permission from ref. 89, Copyright 2014, WILEY-VCH Verlag GmbH \& Co. KGaA, Weinheim.
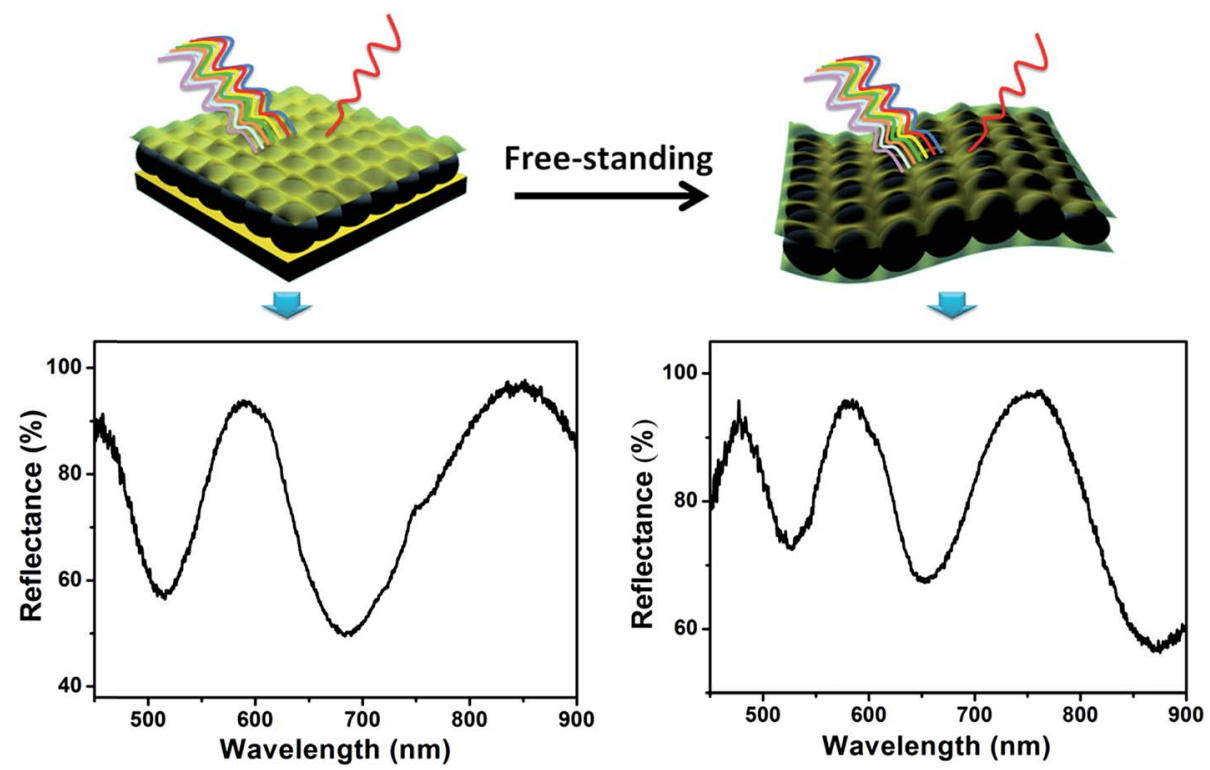

Fig. 17 Poly(N-isopropylacrylamide-co-acrylic acid) (pNIPAm-co-AAc) microgel-based free-standing etalons that exhibit optical properties similar to substrate bound devices. Reprinted with permission from ref. 90, Copyright 2014, Royal Society of Chemistry. 
a

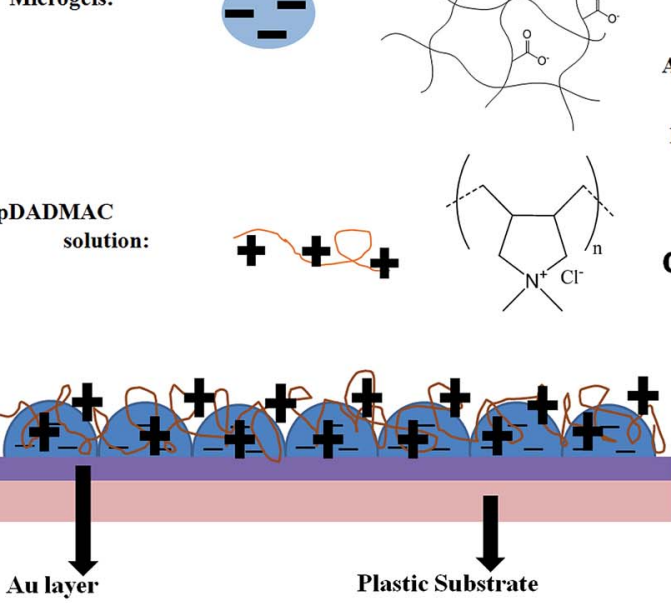

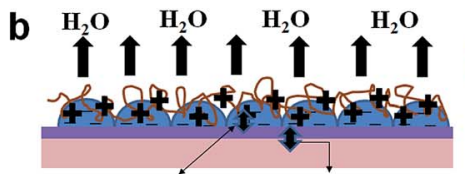

Au-microgel interactions Au-substrate interactions

Decrease humidity \Increase humidity
Hydrophobic interactions

between pDADMAC

Electrostatic interactions between

microgel and pDADMAC
C

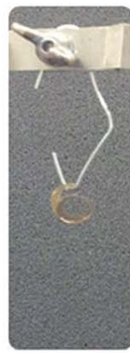

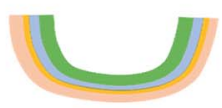

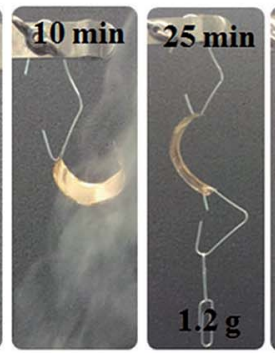

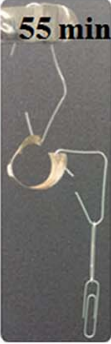
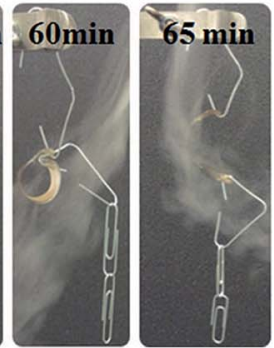

Fig. 18 (a) Polymer-based muscles were constructed by depositing a single layer of pNIPAm-co-AAc microgels on a flexible plastic substrate (bottom layer) coated with a $\mathrm{Au} / \mathrm{Cr}$ layer. After adding a solution of pDADMAC on top of the layer, strong electrostatic interactions between the negatively charged microgels and the positively charged pDADMAC were formed. (b) As the environmental humidity is varied, the pDADMAC layer becomes hydrated/dehydrated, causing it to swell/contract, respectively; the device as a whole unbends or bends, respectively. This bending/unbending mechanism is completely reversible over many cycles. (c) A polymer-based muscle can lift up to $14 \times$ it's own mass. Reprinted with permission from ref. 91, Copyright 2013, ref. 92, Copyright 2014, WILEY-VCH Verlag GmbH \& Co. KGaA, Weinheim.

recent example, our group constructed etalons from microgels that were modified with triphenylmethane leucohydroxide (TPL), the structure and various responses are shown schematically in Fig. 15. TPL is a small molecule that is capable of changing chemistry in response to UV and visible light, $\mathrm{pH}$, temperature and diethylcyanophosphate (GAS, a mimic of the toxic compound tabun). Hence, TPL's incorporation into microgels renders them, and etalons constructed from them, responsive to all the indicated stimuli. ${ }^{89}$ The data for the etalons responding to multiple stimuli are shown in Fig. 16. When UV light was exposed to the devices, the peaks in the reflectance spectrum red shift due to TPL ionization, which yields Coulombic repulsion (and osmotic effects) in the polymer network and hence swelling. The device optical properties return to the original state when the etalons were stored in the dark. The devices also respond to solution $\mathrm{pH}$ changes by becoming ionized/neutralized over the $\mathrm{pH}$ range of $2-8$. The devices also show a response to the presence of GAS - when the microgels are in the presence of tabun, they are ionized. Finally, laser light can be used to stimulate the TPL, which absorbs at $633 \mathrm{~nm}$. When the molecule relaxes it releases heat, which can raise the local temperature in the device, leading to microgel collapse and a blue shift in the device's reflectance spectrum.

\section{Future}

While we have shown that microgel-based etalons can be fabricated on various planar substrates, there are some substrates that are difficult to coat with etalons, e.g., curved surfaces, rods, and tubes. Furthermore, for our sensing and monitoring efforts, it is advantageous to fabricate devices that can be adhered to skin. To achieve this, it would be beneficial to fabricate microgel-based etalons on planar substrates, followed by their desorption. The desorbed etalon can then be adhered to any other substrate as needed. To demonstrate that this is possible, we fabricated free-standing pNIPAm-co-AAc microgelbased etalons that exhibit high quality optical properties, which are capable of being transferred to multiple substrates, ${ }^{90}$ as shown in Fig. 17. The desorbed etalons exhibit similar optical properties to the substrate adhered etalons, and retain their $\mathrm{pH}$ and temperature responsivity. This free-standing optical device will open new applications for sensing in environments that cannot tolerate the planar etalon geometry. The skin-adhered devices can also be used for real-time monitoring of human/ animal health.

Future sensing platforms from our group will also exploit the function of polymer-based muscles we have developed. ${ }^{91-93}$ The structure and function of the devices is shown in Fig. 18. Like the etalons, the devices are constructed by adding a monolayer of negatively charged microgels onto a Au-coated substrate (in this case plastic). A solution of a cationic polyelectrolyte, poly(diallyldimethyl ammonium chloride) (pDADMAC) was subsequently added onto the microgel layer and allowed to dry (at low humidity). The layer depositions are done with solutions that have pHs that promote strong electrostatic interactions between the negatively charged microgels and the positively charged PDADMAC. Therefore, when the PDADMAC layer dries and contracts, the plastic bends. Subsequently, if the environmental humidity is increased, the pDADMAC layer rehydrates, 
a

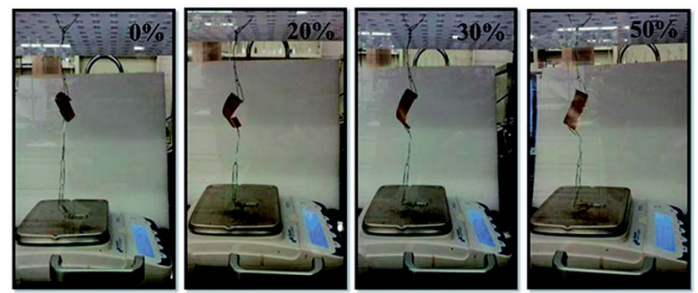

b

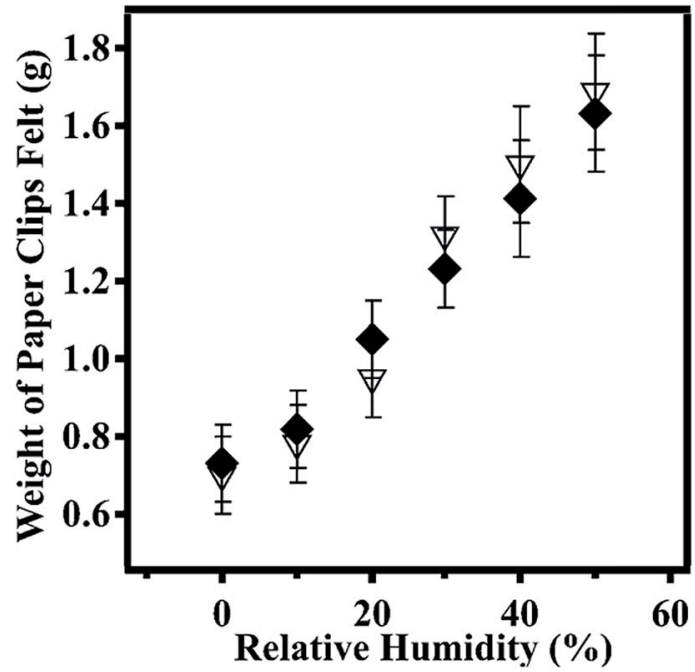

Fig. 19 (a) A device, holding weights, is suspended over a balance; the weights contacting the balance pan. As the humidity was increased, the pDADMAC layer swells, causing the film to open up, adding more of the paperclips to the balance pan and subsequently increasing the measured mass, and vice versa. (b) The measured mass of the paperclips on the balance pan as a function of $(\bullet)$ increasing and $(\nabla)$ decreasing humidity. Reprinted with permission from ref. 93, Copyright 2014, Royal Society of Chemistry.

and the device unbends, as shown in Fig. 18(b) and (c). This bending/unbending mechanism is completely reversible over many cycles. We further showed that the devices can be used to lift weights and are "pound-for-pound" stronger that human arms.

In a follow up investigation, we showed that this behavior could be used to sense atmospheric humidity. ${ }^{93}$ That is, by hanging weights from the polymer-based muscles, and allowing the weights to contact a balance pan, the mass felt by the balance could be correlated to atmospheric humidity, as shown in Fig. 19. This polymer-based muscle motif will be used for future sensing applications.

\section{Conclusion}

The versatility of pNIPAm microgel syntheses allows microgels with a variety of responsivities to be generated. Since our discovery of microgel-based etalons, optical devices, which exhibit optical properties that depend on multiple stimuli could be generated. As we have shown, these optical devices have found numerous sensing applications. Specifically, we demonstrated that etalons could be constructed that respond to temperature, $\mathrm{pH}$, light, electric field, glucose, protein and DNA. This technology is extremely easy to work with, and the devices are simple to fabricate. Furthermore, the technology is inexpensive - a typical device that can be used for $\mathrm{pH}$ sensing cost pennies, and are reusable. Therefore, this optical device has great potential for future applications.

\section{Acknowledgements}

MJS acknowledges funding from the University of Alberta (the Department of Chemistry and the Faculty of Science), the Natural Sciences and Engineering Research Council of Canada (NSERC), the Canada Foundation for Innovation (CFI), the Alberta Advanced Education \& Technology Small Equipment Grants Program (AET/SEGP), Grand Challenges Canada and ICIMPACTS. YG and XL acknowledge Alberta Innovates Technology Futures (AITF) for graduate student scholarships.

\section{References}

1 I. C. Kwon, Y. H. Bae and S. W. Kim, Electrically credible polymer gel for controlled release of drugs, Nature, 1991, 354, 291-293.

2 A. Suzuki and T. Tanaka, Phase transition in polymer gels induced by visible light, Nature, 1990, 346, 345-347.

3 R. P. Sijbesma, F. H. Beijer, L. Brunsveld, B. J. Folmer, J. K. Hirschberg, R. F. Lange, J. K. Lowe and E. Meijer, Reversible polymers formed from self-complementary monomers using quadruple hydrogen bonding, Science, 1997, 278, 1601-1604.

4 M. A. C. Stuart, W. T. Huck, J. Genzer, M. Müller, C. Ober, M. Stamm, G. B. Sukhorukov, I. Szleifer, V. V. Tsukruk and M. Urban, Emerging applications of stimuli-responsive polymer materials, Nat. Mater., 2010, 9, 101-113.

5 S.-K. Ahn, R. M. Kasi, S.-C. Kim, N. Sharma and Y. Zhou, Stimuli-responsive polymer gels, Soft Matter, 2008, 4, 11511157.

6 C. de las Heras Alarcón, S. Pennadam and C. Alexander, Stimuli responsive polymers for biomedical applications, Chem. Soc. Rev., 2005, 34, 276-285.

7 M. Heskins and J. E. Guillet, Solution properties of $\operatorname{poly}(\mathrm{N}$ isopropylacrylamide), J. Macromol. Sci., Chem., 1968, 2, 1441-1455.

8 H. G. Schild, M. Muthukumar and D. A. Tirrell, Cononsolvency in mixed aqueous solutions of $\operatorname{poly}(N$ isopropylacrylamide), Macromolecules, 1991, 24, 948-952.

9 F. Ganachaud, M. J. Monteiro, R. G. Gilbert, M.-A. Dourges, S. H. Thang and E. Rizzardo, Molecular weight characterization of poly( $N$-isopropylacrylamide) prepared by living free-radical polymerization, Macromolecules, 2000, 33, 6738-6745.

10 R. Plummer, D. J. Hill and A. K. Whittaker, Solution properties of star and linear poly( $N$-isopropylacrylamide), Macromolecules, 2006, 39, 8379-8388.

11 J. Ye, J. Xu, J. Hu, X. Wang, G. Zhang, S. Liu and C. Wu, Comparative study of temperature-induced association of cyclic and linear poly( $N$-isopropylacrylamide) chains in dilute solutions by laser light scattering and stopped-flow temperature jump, Macromolecules, 2008, 41, 4416-4422. 
12 R. Pelton and P. Chibante, Preparation of aqueous latices with $N$-isopropylacrylamide, Colloids Surf., 1986, 20, 247-256.

13 R. Pelton, Temperature-sensitive aqueous microgels, Adv. Colloid Interface Sci., 2000, 85, 1-33.

14 C. D. Jones and L. A. Lyon, Synthesis and characterization of multiresponsive core-shell microgels, Macromolecules, 2000, 33, 8301-8306.

15 A. S. Hoffman, Hydrogels for biomedical applications, Adv. Drug Delivery Rev., 2002, 54, 3-12.

16 J. Chen, H. Park and K. Park, Synthesis of superporous hydrogels: hydrogels with fast swelling and superabsorbent properties, J. Biomed. Mater. Res., 1999, 44, 53-62.

17 R. A. Stile, W. R. Burghardt and K. E. Healy, Synthesis and characterization of injectable poly( $N$-isopropylacrylamide)based hydrogels that support tissue formation in vitro, Macromolecules, 1999, 32, 7370-7379.

18 H. Kawaguchi, M. Kawahara, N. Yaguchi, F. Hoshino and Y. Ohtsuka, Hydrogel microspheres I. Preparation of monodisperse hydrogel microspheres of submicron or micron size, Polym. J., 1988, 20, 903-909.

19 M. Ballauff and Y. Lu, "Smart" nanoparticles: preparation, characterization and applications, Polymer, 2007, 48, 18151823.

$20 \mathrm{H}$. Senff and W. Richtering, Temperature sensitive microgel suspensions: Colloidal phase behavior and rheology of soft spheres, J. Chem. Phys., 1999, 111, 1705-1711.

21 T. R. Hoare and D. S. Kohane, Hydrogels in drug delivery: progress and challenges, Polymer, 2008, 49, 1993-2007.

$22 \mathrm{C}$. Wu and S. Zhou, Light scattering study of spherical poly( $N$-isopropylacrylamide) microgels, J. Macromol. Sci., Part B: Phys., 1997, 36, 345-355.

23 T. Liu, J. Hu, J. Yin, Y. Zhang, C. Li and S. Liu, Enhancing detection sensitivity of responsive microgel-based $\mathrm{Cu}$ (II) chemosensors via thermo-induced volume phase transitions, Chem. Mater., 2009, 21, 3439-3446.

24 R. Contreras-Cáceres, A. Sánchez-Iglesias, M. Karg, I. Pastoriza-Santos, J. Pérez-Juste, J. Pacifico, T. Hellweg, A. Fernández-Barbero and L. M. Liz-Marzán, Encapsulation and growth of gold nanoparticles in thermoresponsive microgels, Adv. Mater., 2008, 20, 1666-1670.

25 S. Su, M. M. Ali, C. D. Filipe, Y. Li and R. Pelton, Microgelbased inks for paper-supported biosensing applications, Biomacromolecules, 2008, 9, 935-941.

26 D. Parasuraman and M. J. Serpe, $\operatorname{Poly}(N$ Isopropylacrylamide) microgel-based assemblies for organic dye removal from water, ACS Appl. Mater. Interfaces, 2011, 3, 4714-4721.

27 D. Parasuraman, E. Leung and M. J. Serpe, Poly $(N-$ isopropylacrylamide) microgel based assemblies for organic dye removal from water: microgel diameter effects, Colloid Polym. Sci., 2012, 290, 1053-1064.

28 Y. Guan and Y. Zhang, PNIPAM microgels for biomedical applications: From dispersed particles to 3D assemblies, Soft Matter, 2011, 7, 6375-6384.

29 J. K. Oh, R. Drumright, D. J. Siegwart and K. Matyjaszewski, The development of microgels/nanogels for drug delivery applications, Prog. Polym. Sci., 2008, 33, 448-477.
30 Y. Gao, G. P. Zago, Z. Jia and M. J. Serpe, Controlled and Triggered Small Molecule Release from a Confined Polymer Film, ACS Appl. Mater. Interfaces, 2013, 5, 98039808.

$31 \mathrm{~S}$. Tsuji and H. Kawaguchi, Self-assembly of $\operatorname{poly}(N-$ isopropylacrylamide)-carrying microspheres into twodimensional colloidal arrays, Langmuir, 2005, 21, 2434-2437.

32 T. Hoare and R. Pelton, Highly $\mathrm{pH}$ and temperature responsive microgels functionalized with vinylacetic acid, Macromolecules, 2004, 37, 2544-2550.

33 J. D. Debord and L. A. Lyon, Synthesis and characterization of pH-responsive copolymer microgels with tunable volume phase transition temperatures, Langmuir, 2003, 19, 7662-7664.

34 B. Brugger and W. Richtering, Magnetic, Thermosensitive Microgels as Stimuli-Responsive Emulsifiers Allowing for Remote Control of Separability and Stability of Oil in Water-Emulsions, Adv. Mater., 2007, 19, 2973-2978.

35 S. Bhattacharya, F. Eckert, V. Boyko and A. Pich, Temperature-, $\mathrm{pH}-$, and Magnetic-Field-Sensitive Hybrid Microgels, Small, 2007, 3, 650-657.

36 Z. Meng, M. H. Smith and L. A. Lyon, Temperatureprogrammed synthesis of micron-sized multi-responsive microgels, Colloid Polym. Sci., 2009, 287, 277-285.

37 J. Zhang, S. Xu and E. Kumacheva, Polymer microgels: reactors for semiconductor, metal, and magnetic nanoparticles, J. Am. Chem. Soc., 2004, 126, 7908-7914.

38 D. Palioura, S. Armes, S. Anastasiadis and M. Vamvakaki, Metal nanocrystals incorporated within $\mathrm{pH}$-responsive microgel particles, Langmuir, 2007, 23, 5761-5768.

39 I. Gorelikov, L. M. Field and E. Kumacheva, Hybrid microgels photoresponsive in the near-infrared spectral range, J. Am. Chem. Soc., 2004, 126, 15938-15939.

40 T. Hoare and R. Pelton, Charge-switching, amphoteric glucose-responsive microgels with physiological swelling activity, Biomacromolecules, 2008, 9, 733-740.

41 V. Lapeyre, I. Gosse, S. Chevreux and V. Ravaine, Monodispersed glucose-responsive microgels operating at physiological salinity, Biomacromolecules, 2006, 7, 3356-3363.

42 C. D. Sorrell and M. J. Serpe, Glucose sensitive $\operatorname{poly}(N$ isopropylacrylamide) microgel based etalons, Anal. Bioanal. Chem., 2012, 402, 2385-2393.

43 M. Karg, I. Pastoriza-Santos, B. Rodriguez-Gonzalez, R. von Klitzing, S. Wellert and T. Hellweg, Temperature, pH, and ionic strength induced changes of the swelling behavior of PNIPAM- poly(allylacetic acid) copolymer microgels, Langmuir, 2008, 24, 6300-6306.

44 T. Gan, Y. Zhang and Y. Guan, In situ gelation of P (NIPAMHEMA) microgel dispersion and its applications as injectable 3D cell scaffold, Biomacromolecules, 2009, 10, 1410-1415.

$45 \mathrm{H}$. Chen and L. L. Dai, Adsorption and release of active species into and from multifunctional ionic microgel particles, Langmuir, 2013, 29, 11227-11235.

46 V. Lapeyre, C. Ancla, B. Catargi and V. Ravaine, Glucoseresponsive microgels with a core-shell structure, J. Colloid Interface Sci., 2008, 327, 316-323. 
47 J. H. Holtz and S. A. Asher, Polymerized colloidal crystal hydrogel films as intelligent chemical sensing materials, Nature, 1997, 389, 829-832.

48 J. M. Weissman, H. B. Sunkara, S. T. Albert and S. A. Asher, Thermally switchable periodicities and diffraction from mesoscopically ordered materials, Science, 1996, 274, 959963.

49 C. E. Reese, A. V. Mikhonin, M. Kamenjicki, A. Tikhonov and S. A. Asher, Nanogel nanosecond photonic crystal optical switching, J. Am. Chem. Soc., 2004, 126, 1493-1496.

50 N. L. Smith, Z. Hong and S. A. Asher, Responsive ionic liquid-polymer 2D photonic crystal gas sensors, Analyst, 2014, 139, 6379-6386.

51 J.-T. Zhang, N. Smith and S. A. Asher, Two-dimensional photonic crystal surfactant detection, Anal. Chem., 2012, 84, 6416-6420.

52 M. M. Ward Muscatello, L. E. Stunja and S. A. Asher, Polymerized crystalline colloidal array sensing of high glucose concentrations, Anal. Chem., 2009, 81, 4978-4986.

53 J. P. Walker and S. A. Asher, Acetylcholinesterase-based organophosphate nerve agent sensing photonic crystal, Anal. Chem., 2005, 77, 1596-1600.

54 X. Xu, A. V. Goponenko and S. A. Asher, Polymerized polyHEMA photonic crystals: $\mathrm{pH}$ and ethanol sensor materials, J. Am. Chem. Soc., 2008, 130, 3113-3119.

55 Z. Cai, J.-T. Zhang, F. Xue, Z. Hong, D. Punihaole and S. A. Asher, 2D Photonic Crystal Protein Hydrogel Coulometer for Sensing Serum Albumin Ligand Binding, Anal. Chem., 2014, 86, 4840-4847.

56 M. C. Chiappelli and R. C. Hayward, Photonic Multilayer Sensors from Photo-Crosslinkable Polymer Films, Adv. Mater., 2012, 24, 6100-6104.

57 H. Wang and K.-Q. Zhang, Photonic crystal structures with tunable structure color as colorimetric sensors, Sensors, 2013, 13, 4192-4213.

58 M. Giese, L. K. Blusch, M. K. Khan, W. Y. Hamad and M. J. MacLachlan, Responsive Mesoporous Photonic Cellulose Films by Supramolecular Cotemplating, Angew. Chem., Int. Ed., 2014, 53, 8880-8884.

59 J. Wang, Y. Hu, R. Deng, R. Liang, W. Li, S. Liu and J. Zhu, Multiresponsive Hydrogel Photonic Crystal Microparticles with Inverse-Opal Structure, Langmuir, 2013, 29, 8825-8834.

60 E. Tian, J. Wang, Y. Zheng, Y. Song, L. Jiang and D. Zhu, Colorful humidity sensitive photonic crystal hydrogel, $J$. Mater. Chem., 2008, 18, 1116-1122.

61 L. Hu, A. K. Sarker, M. R. Islam, X. Li, Z. Lu and M. J. Serpe, Poly( $N$-isopropylacrylamide) microgel-based assemblies, $J$. Polym. Sci., Part A: Polym. Chem., 2013, 51, 3004-3020.

$62 \mathrm{~L}$. Hu and M. J. Serpe, Color-tunable etalons assembled from Poly( $N$-isopropylacrylamide) based microgels, Polymers, 2012, 4, 134-149.

63 M. R. Islam, A. Ahiabu, X. Li and M. J. Serpe, Poly $(N-$ isopropylacrylamide) Microgel-Based Optical Devices for Sensing and Biosensing, Sensors, 2014, 14, 8984-8995.

$64 \mathrm{~L}$. Hu and J. Michael, Poly( $N$-Isopropylacrylamide) MicrogelBased Etalons for Optical Sensing, J. Anal. Bioanal. Tech., 2012, 3, 132.
65 A. C. Arsenault, D. P. Puzzo, I. Manners and G. A. Ozin, Photonic-crystal full-colour displays, Nat. Photonics, 2007, 1, 468-472.

66 J. D. Debord and L. A. Lyon, Thermoresponsive photonic crystals, J. Phys. Chem. B, 2000, 104, 6327-6331.

67 Y. J. Lee and P. V. Braun, Tunable inverse opal hydrogel pH sensors, Adv. Mater., 2003, 15, 563-566.

68 Y. Kang, J. J. Walish, T. Gorishnyy and E. L. Thomas, Broadwavelength-range chemically tunable block-copolymer photonic gels, Nat. Mater., 2007, 6, 957-960.

69 M. R. Islam, Z. Lu, X. Li, A. K. Sarker, L. Hu, P. Choi, X. Li, N. Hakobyan and M. J. Serpe, Responsive polymers for analytical applications: A review, Anal. Chim. Acta, 2013, 789, 17-32.

70 M. R. Islam and M. J. Serpe, Polyelectrolyte mediated intra and intermolecular crosslinking in microgel-based etalons for sensing protein concentration in solution, Chem. Commun., 2013, 49, 2646-2648.

71 M. R. Islam and M. J. Serpe, Label-free detection of low protein concentration in solution using a novel colorimetric assay, Biosens. Bioelectron., 2013, 49, 133-138.

72 M. R. Islam and M. J. Serpe, Poly( $N$-isopropylacrylamide) microgel-based etalons and etalon arrays for determining the molecular weight of polymers in solution, APL Mater., 2013, 1, 052108.

$73 \mathrm{~L} . \mathrm{Hu}$ and M. J. Serpe, Color modulation of spatially isolated regions on a single poly( $N$-isopropylacrylamide) microgel based etalon, J. Mater. Chem., 2012, 22, 8199-8202.

74 L. Hu and M. J. Serpe, The Influence of Deposition Solution $\mathrm{pH}$ and Ionic Strength on the Quality of $\operatorname{Poly}(\mathrm{N}$ isopropylacrylamide) Microgel-Based Thin Films and Etalons, ACS Appl. Mater. Interfaces, 2013, 5, 11977-11983.

75 C. D. Sorrell, M. C. Carter and M. J. Serpe, Color Tunable Poly(N-Isopropylacrylamide)-co-Acrylic Acid Microgel-Au Hybrid Assemblies, Adv. Funct. Mater., 2011, 21, 425-433.

76 C. D. Sorrell and M. J. Serpe, Reflection Order Selectivity of Color-Tunable Poly( $N$-isopropylacrylamide) Microgel Based Etalons, Adv. Mater., 2011, 23, 4088-4092.

$77 \mathrm{~L}$. $\mathrm{Hu}$ and M. J. Serpe, Controlling the response of color tunable poly( $N$-isopropylacrylamide) microgel-based etalons with hysteresis, Chem. Commun., 2013, 49, 26492651.

78 M. C. Carter, C. D. Sorrell and M. J. Serpe, Deswelling kinetics of color tunable poly $(N$-isopropylacrylamide) microgel-based Etalons, J. Phys. Chem. B, 2011, 115, 1435914368.

79 I. N. Heppner and M. J. Serpe, Poly( $N$-isopropylacrylamide) microgel-based etalons constructed from various metal layers, Colloid Polym. Sci., 2013, 291, 1557-1562.

80 C. D. Sorrell, M. C. Carter and M. J. Serpe, A "paint-on" protocol for the facile assembly of uniform microgel coatings for color tunable etalon fabrication, ACS Appl. Mater. Interfaces, 2011, 3, 1140-1147.

81 K. C. Johnson, F. Mendez and M. J. Serpe, Detecting solution $\mathrm{pH}$ changes using poly( $\mathrm{N}$-isopropylacrylamide)-co-acrylic acid microgel-based etalon modified quartz crystal microbalances, Anal. Chim. Acta, 2012, 739, 83-88. 
82 M. R. Islam, K. C. Johnson and M. J. Serpe, Microgel-based etalon coated quartz crystal microbalances for detecting solution pH: The effect of Au overlayer thickness, Anal. Chim. Acta, 2013, 792, 110-114.

83 Y. Gao and M. J. Serpe, Light-Induced Color Changes of Microgel-Based Etalons, ACS Appl. Mater. Interfaces, 2014, 6, 8461-8466.

84 Q. M. Zhang, X. Li, M. R. Islam, M. Wei and M. J. Serpe, Light switchable optical materials from azobenzene crosslinked poly( $N$-isopropylacrylamide)-based microgels, J. Mater. Chem. C, 2014, 2, 6961-6965.

$85 \mathrm{~W}$. Xu, Y. Gao and M. J. Serpe, Electrochemically color tunable poly( $N$-isopropylacrylamide $)$ microgel-based etalons, J. Mater. Chem. C, 2014, 2, 3873-3878.

86 M. R. Islam and M. J. Serpe, Polymer-based devices for the label-free detection of DNA in solution: low DNA concentrations yield large signals, Anal. Bioanal. Chem., 2014, 1-7.

87 M. R. Islam and M. J. Serpe, A novel label-free colorimetric assay for DNA concentration in solution, Anal. Chim. Acta, 2014, 843, 83-88.
88 M. R. Islam and M. J. Serpe, Penetration of polyelectrolytes into charged poly( $N$-isopropylacrylamide) microgel layers confined between two surfaces, Macromolecules, 2013, 46, 1599-1606.

89 Q. M. Zhang, W. Xu and M. J. Serpe, Optical Devices Constructed from Multiresponsive Microgels, Angew. Chem., Int. Ed., 2014, 126, 4927-4931.

$90 \mathrm{Y}$. Gao, W. Xu and M. J. Serpe, Free-standing $\operatorname{poly}(N-$ isopropylacrylamide) microgel-based etalons, J. Mater. Chem. C, 2014, 2, 5878-5884.

91 M. R. Islam, X. Li, K. Smyth and M. J. Serpe, Polymer-Based Muscle Expansion and Contraction, Angew. Chem., Int. Ed., 2013, 52, 10330-10333.

$92 \mathrm{X}$. Li and M. J. Serpe, Understanding and Controlling the Self-Folding Behavior of Poly( $N$-Isopropylacrylamide) Microgel-Based Devices, Adv. Funct. Mater., 2014, 24, 41194126.

93 M. R. Islam and M. J. Serpe, Poly( $N$-isopropylacrylamide) microgel-based thin film actuators for humidity sensing, RSC Adv., 2014, 4, 31937-31940. 